\title{
Editorial
}

\section{RCHOT ahora bilingüe: Un gran paso al futuro}

\author{
Julio Urrutia ${ }^{1,2}$ \\ 1 Departamento de Ortopedia y Traumatología, Escuela de Medicina, \\ Pontificia Universidad Católica de Chile, Santiago, Chile \\ 2 Presidente, Sociedad Chilena de Ortopedia y Traumatología, \\ Santiago, Chile
}

Rev Chil Ortop Traumatol 2021;62:1.

La Revista de la Sociedad Chilena de Ortopedia y Traumatología (RCHOT) es la publicación oficial de la Sociedad Chilena de Ortopedia y Traumatología desde 1953, y como tal, comunica los trabajos originales, reportes de casos y revisiones de temas de la especialidad de traumatólogos, médicos en formación y de otras especialidades de nuestra comunidad nacional. Desde el año 2017, la RCHOT pasó a publicarse en formato online, y eso ha aumentado su visibilidad internacional, lo que ha permitido recibir trabajos de autores de diversos países hispanoamericanos.

A partir de este número, tenemos la satisfacción de iniciar una edición bilingüe de nuestra revista, en castellano e inglés; esto nos permitirá avanzar en su internacionalización, pues ya no solo será un medio de difusión atractivo para autores hispanoamericanos, sino para autores que busquen una revista abierta a la comunidad global. Este gran avance en nuestra revista ha sido producto de años de trabajo del Comité Editorial de la RCHOT, apoyado por el Directorio de la Sociedad Chilena de Ortopedia y Traumatología, que buscaron tener una plataforma con una Editorial Internacional de prestigio como Thieme. Esperamos que este avance nos permita, en un futuro cercano, alcanzar la esperada indexación de nuestra revista y, más tarde, lograr que ella cuente con Factor de Impacto.

En este apoyo por parte de la Sociedad Chilena de Ortopedia y Traumatología, ha habido un esfuerzo económico para publicar una revista Open Access a través de Thieme, pero también un apoyo directo a múltiples socios y sus grupos de trabajo quienes, a través de los premios del Comité de Investigación, han podido tener fondos para realizar su investigación. Así mismo, el Directorio de la Sociedad Chilena de Ortopedia y Traumatología ha definido múltiples formas de estimular la publicación de los mejores trabajos presentados en nuestro Congreso Anual, de forma que nuestra revista refleje lo mejor de la productividad científica nacional de nuestra especialidad.

Junto con felicitar al Comité Editorial, quiero hacer un especial reconocimiento al Director de la RCHOT, Dr. David Figueroa, por el gran avance que le ha dado a nuestra revista, y que se ve reflejado en éste, nuestro primer número bilingüe. Finalmente, hago un llamado a todos nuestros socios, médicos en formación y profesionales afines a nuestra especialidad a seguir enviando sus comunicaciones a la RCHOT, nuestra revista.
Dirección para correspondencia Julio Urrutia, Departamento de Ortopedia y Traumatología, Escuela de Medicina, Pontificia Universidad Católica de Chile, Chile

(e-mail: jurrutia@med.puc.cl).
DOI https://doi.org/ $10.1055 / \mathrm{s}-0041-1728742$. ISSN $0716-4548$. (c) 2021. Sociedad Chilena de Ortopedia y Traumatologia. All rights reserved.

This is an open access article published by Thieme under the terms of the Creative Commons Attribution-NonDerivative-NonCommercial-License, permitting copying and reproduction so long as the original work is given appropriate credit. Contents may not be used for commercial purposes, or adapted, remixed, transformed or built upon. (https://creativecommons.org/ licenses/by-nc-nd/4.0/)

Thieme Revinter Publicações Ltda., Rua do Matoso 170, Rio de Janeiro, RJ, CEP 20270-135, Brazil 